\title{
Cartilage of the patella
}

\section{Topographical variation of glycosaminoglycan content in normal and fibrillated tissue}

\author{
C. FICAT* AND A. MAROUDAS \\ From the Department of Mechanical Engineering, Imperial College, London SW 7
}

Ficat, C., and Maroudas, A. (1975). Annals of the Rheumatic Diseases, 34, 515-519. Cartilage of the patella. Topographical variation of glycosaminoglycan content in normal and fibrillated tissue. The variation in the glycosaminoglycan content was studied at different sites of the patella, both where the cartilage was intact and where it showed varying degrees of fibrillation. It was found that when the cartilage surface was intact the glycosaminoglycan content was the same at the different sites of the patella.

Local fibrillation always gave rise to a local lowering of fixed charge density, the magnitude of the latter correlating well with the visual assessment of the severity of the lesion. Even in the presence of severe lesions, if there was a site left on the patella where the cartilage was visually normal, its fixed charge density was also at a normal level. Thus, the depletion of glycosaminoglycans is a local phenomenon, limited to the area of fibrillation.

The glycosaminoglycan content of normal cartilage is lower in the knee joint than in the hip. This fact, together with the existence of high pressures during load bearing, may be responsible for the greater frequency of destructive lesions affecting the cartilage of the patella compared with that of the hip.

The age-related lesions affecting the articular cartilage of the patella have been investigated recently in great detail with respect to their morphology and topography (Emery and Meachim, 1973; Meachim and Emery, 1974; Ficat, 1974).

Degenerative changes of the patella cartilage appear to be related to various localized anatomical and mechanical factors. Thus, on the lateral facet the lesions appearing at the junction of the median vertical ridge and the transverse horizontal ridge are caused by high local pressure (Ficat, 1973). It is noteworthy that this is also the region where cartilage is thicker than on any other joint surface. On the medial facet, on the other hand, lesions seem to result from incongruence. The medial facet is convex, with a vertical or an oblique ridge running through it. During the movements of flexionextension this region comes into contact with a line of ridges with aggressive features on the medial condyle (Ficat, 1974) and is subject as a result to a destructive 'ploughing' action.

We present a study of the glycosaminoglycan content at different sites on the patella both in cases where the cartilage surface was normal and also where it exhibited varying degrees of fibrillation. Since the cartilage of the patella seems to show destructive changes more frequently than that of other joints, it was thought of interest to find out whether there were any inherent biochemical features in normal, intact cartilage which might predispose it to these changes.

The fixed charge density method of estimating the total glycosaminoglycan content was used. This had been previously used by one of us to examine topographical variations in the cartilage of the hip joint (Maroudas and Thomas, 1970; Maroudas, Evans, and Almeida, 1973). The advantage of this method is that it is rapid, accurate, and can be used on very small quantities of tissue.

\section{Materials and methods}

Forty patellae and 22 femoropatellar joints obtained at autopsy from patients aged 14 to 83 years were examined by eye after staining with Indian ink to show the condition of the surface (Meachim, 1972). Most of the patellae, but only a few femoral condyles were subsequently analysed chemically. 
From each patella several full depth cartilage specimens, $0.5 \mathrm{~cm}$ in diameter, were cored out. These were usually taken from both lateral and medial facets. The fixed charge density of these specimens was determined by the tracer cation method as described previously (Maroudas and Thomas, 1970; Maroudas and others, 1973). Measurements were first made on full thickness specimens, some of which were subsequently cut into approximately $300 \mu \mathrm{m}$ slices in order to obtain the variation of the glycosaminoglycan content as a function of the distance from the articular surface.

\section{Results and discussion}

Although no patella in our study was completely free from fibrillation, a few showed minimal surface changes only. A patella such as this with light fibrillation limited to cartilage borders is shown in Fig. $1 A$. It can be seen that the glycosaminoglycan content of specimens taken from the median ridge as well as from the lateral and medial facets is practically identical. The curves of fixed charge density versus depth from the three specimens coincide not only with one another, but also with the curves for the corresponding sites on the femoral trochlea (Fig. 1B).

The above results clearly show that specimens with an intact surface do not differ in their glycosaminoglycan content regardless of from which part of the patella or the opposing trochlea they are taken. Similar conclusions can be reached from results for other patellae. Thus, the Table shows the mean values of fixed charge density for all the cartilage plugs which showed no fibrillation (i.e. no Indian ink uptake at the surface or only minute traces). There was no significant difference between the means of the values for the lateral, medial, or central zones. The overall scatter is relatively low (SD $10 \%$ ).
Table Glycosaminoglycan content (mean $\pm S D$ ) of different sites on the patella. Intact specimens

\begin{tabular}{|c|c|c|c|}
\hline Site & Lateral facet & Central zone & Medial facet \\
\hline $\begin{array}{l}\text { No. of } \\
\text { specimens }\end{array}$ & 12 & 5 & 14 \\
\hline $\begin{array}{l}\text { Fixed charge } \\
\text { density } \\
(\mathrm{mEq} / \mathrm{g})\end{array}$ & $0 \cdot 110 \pm 0 \cdot 010$ & $0 \cdot 105 \pm 0.011$ & $0 \cdot 10^{\prime}$ \\
\hline
\end{tabular}

The number of intact specimens which could be found in the central zone was much more limited than that from the other areas. This observation is consistent with the fact that it is the central zone which is almost constantly subjected to high pressures and is accordingly often the starting point of progressive cartilage lesions (Ficat, 1973).

Patellae such as that shown in Fig. 1, with very little Indian ink staining, are infrequent. The following examples show different types of lesions. Fig. 2 shows a patella with a focus of overt fibrillation at the centre of the medial facet, surrounded by cartilage which is much more superficially fibrillated; the lateral facet has a zone of surface fibrillation, but the area below the transverse ridge is practically intact. The levels of fixed charge density clearly reflect the visually observed state of the cartilage, thus, site MB, showing deep fibrillation with partial thickness loss, has the lowest fixed charge density throughout its depth; sites LA and MA, which are surface fibrillated, also show a considerable lowering of fixed charge density throughout their depth. Sites LB and MC, which are practically free from fibrillation, have the highest fixed charge density.

It is interesting to note that the zone of deep fibrillation on the medial facet corresponds to an

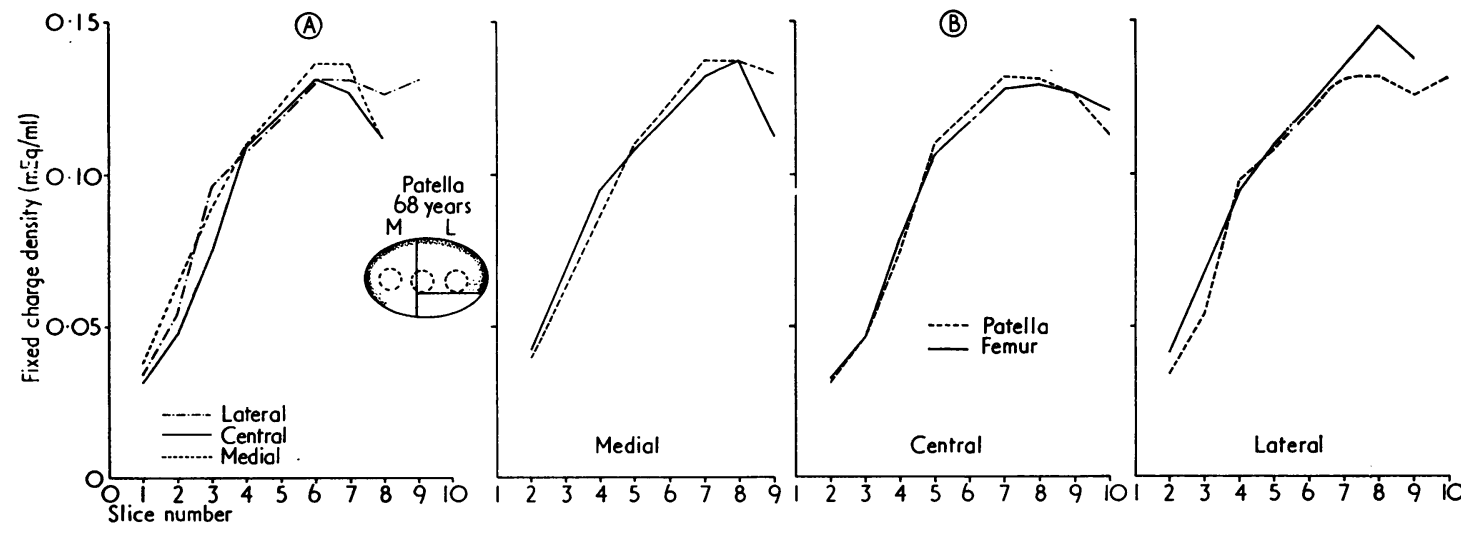

FIG. 1 A Normal patella: curves of fixed charge density versus depth from the articular surface. B Comparison between fixed charge density of the cartilage of the patella and the corresponding sites on the femoral trochlea. Figs. 1-6: degree of shading of articular surface denotes degree of Indian ink uptake and hence degree of fibrillation 


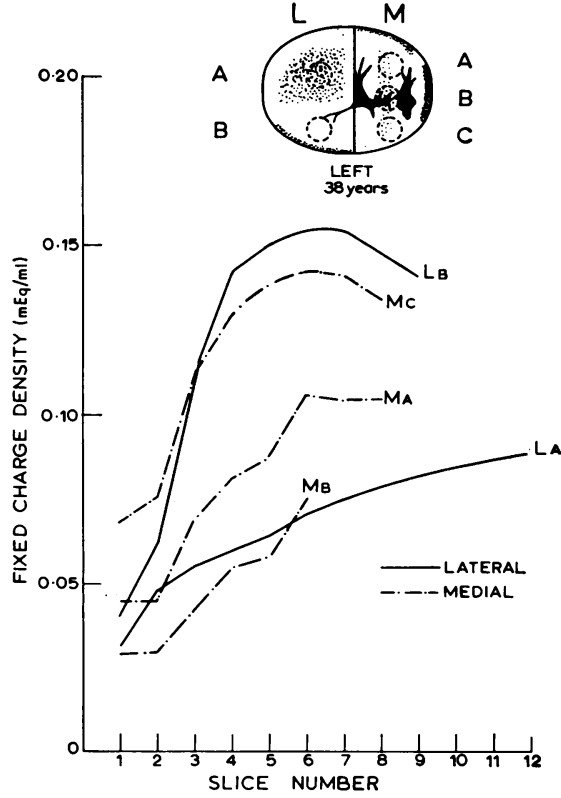

FIG. 2 Curves of fixed charge density versus depth for a patella showing a focus of overt fibrillation on the medial facet

obvious ridge on the femoral condyle which could have caused destruction by a 'ploughing' action.

Unlike the previous example where fibrillation was more pronounced on the medial facet, the patella illustrated in Fig. 3 has a focus of deep fibrillation in the centre of the lateral facet, spreading outwards. The cartilage on the medial facet is intact except for slight fibrillation along the margins. The specimen taken from the fibrillated site (LB) could not be

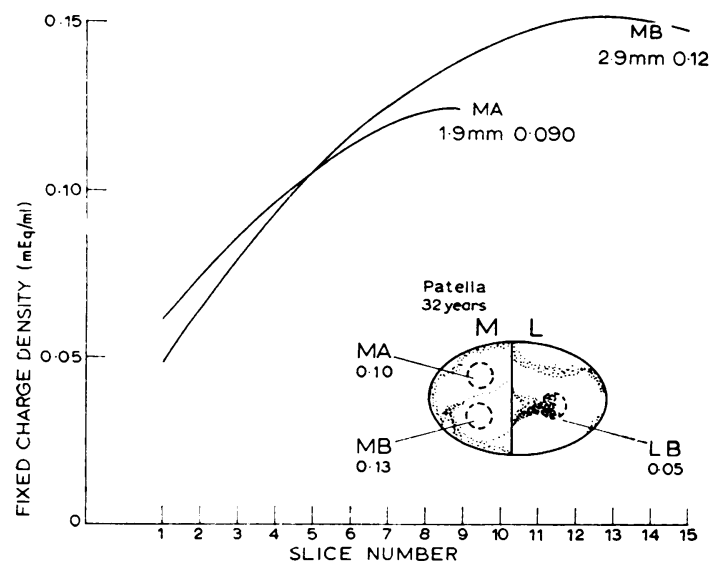

FIG. 3 Curves of fixed charge density versus depth for a patella showing a focus of overt fibrillation on the lateral facet sliced, but its mean fixed charge density was equal to about half the value of the normal specimens on the medial facet. Of the two specimens with intact surfaces, it is the thicker one which has a somewhat higher mean fixed charge density. This appears to be a thickness effect as the curves of fixed charge density versus depth initially coincide; the curve for the thicker specimen rises to higher values in the deep zone. A similar observation has been made on a few other cases. Thus, if normal cartilage specimens differ considerably in thickness from one another, their mean fixed charge density may also differ, because of the variation in the values attained in the deep zone.

Fig. 4 shows a patella in which both the medial and lateral facets have foci of overt fibrillation, and both are situated at the centre of a vertical ridge. Somewhat less deep fibrillation is present along the lateral transverse ridge and also around the cartilage margins. The fixed charge density of the various sites is consistent with the observed degree of fibrillation.

Fig. 5 shows a clear case of osteoarthrosis of the patella, with marginal osteophytes. There is widespread bone exposure and the central transverse band has only a thin layer remaining of very fibrillated cartilage. The fixed charge density at this site is very low $(0.041 \mathrm{mEq} / \mathrm{g})$. The upper band of cartilage shows surface fibrillation and its fixed charge density is much higher $(0.075 \mathrm{mEq} / \mathrm{g})$.

The corresponding femoral trochlea is also fibrillated, but less so than the patella. This appears often to be the case (Emery and Meachim, 1973). There is a very small zone of bone exposure on the medial side of the trochlea, probably caused by the medial osteophyte on the patella. There is also only a relatively localized region of overt fibrillation in the centre, with a fixed charge density of $0.05 \mathrm{mEq} / \mathrm{g}$. Most of the remainder has a fixed charge density around $0.089 \mathrm{mEq} / \mathrm{g}$, with a small area appearing completely intact which has a fixed charge density of $0 \cdot 115 \mathrm{mEq} / \mathrm{g}$; such a value is usually characteristic

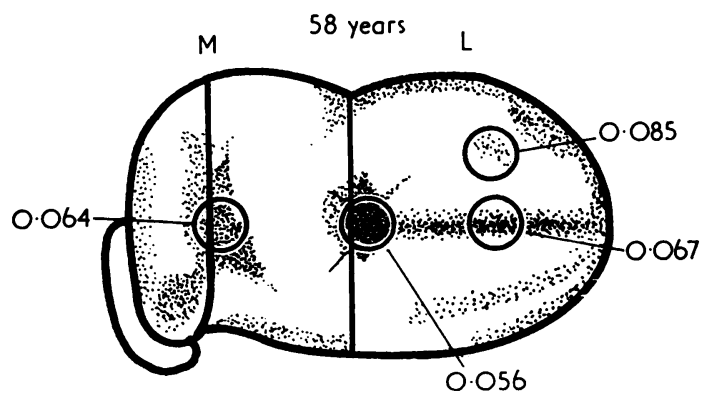

FIG. 4 Fixed charge density at different sites of a patella showing foci of overt fibrillation on both the medial and the lateral facets 


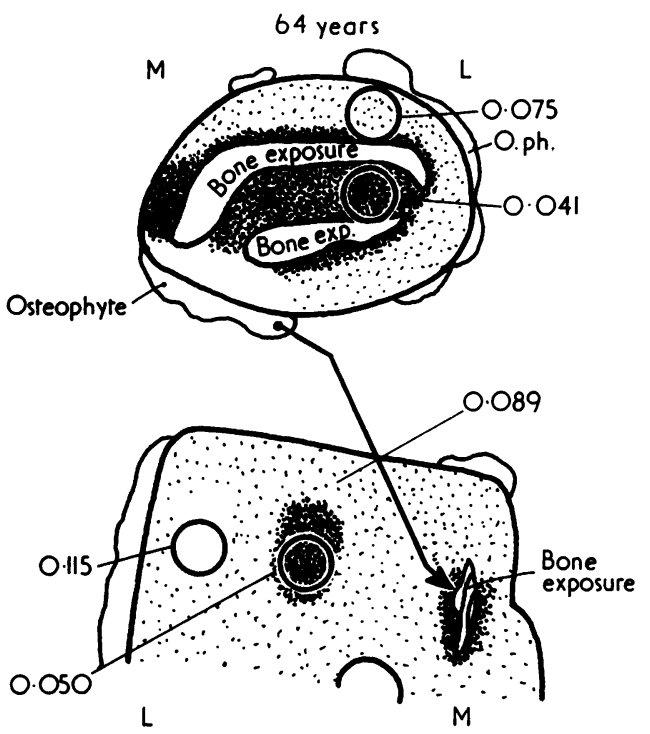

FIG. 5 Example of an osteoarthrotic patella

of completely normal cartilage and this shows that even when a site with an intact surface is close to a fibrillated region, it is still characterized by a normal level of glycosaminoglycans. The same observation was made in the case of the femoral head (Maroudas and others, 1973).

Fig. 6 shows how localized the destructive changes on the patella can be under some circumstances: while the lateral facet is totally denuded, the medial facet is covered with cartilage which looks normal and has a high fixed charge density.

Fig. 7 summarizes the results obtained in the present series in a graph of fixed charge density versus age for both normal and fibrillated specimens.

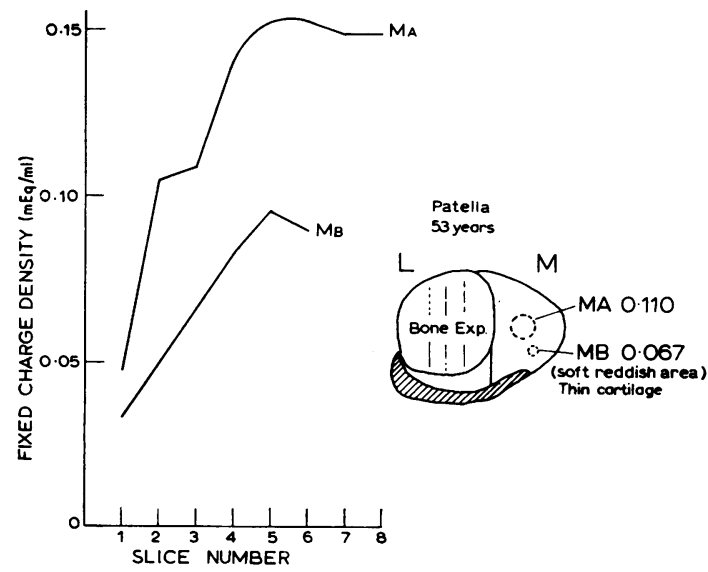

FIG. 6 Example of the localized nature of the destructive changes on the patella

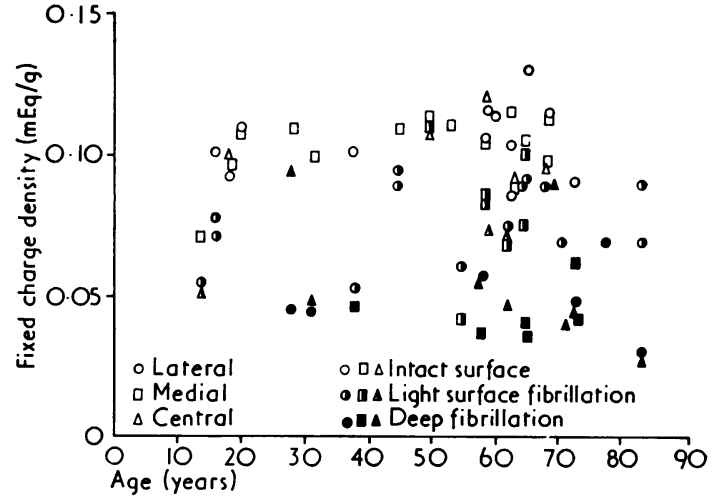

FIG. 7 Graph of fixed charge density versus age for normal and fibrillated specimens from the patella

It can be seen that from the age of 20 years onwards there does not appear to be any variation in the glycosaminoglycan content with age. This is consistent with observations by previous authors (e.g. Miles and Eichelberger, 1964; Anderson, Ludowieg, Harper, and Engleman, 1964; Bollet and Nance, 1966; Maroudas, and others, 1973). Below the age of 20 fixed charge density is lower than in the adult. The same observation was made in the case of hip cartilage (Maroudas and others, 1973).

The mean fixed charge density of specimens showing deep fibrillation is about $0.05 \mathrm{mEq} / \mathrm{g}$, that of superficially fibrillated ones is in the approximate range $0.06-0.09 \mathrm{mEq} / \mathrm{g}$, and normal specimens have a fixed charge density of about $0.11 \mathrm{mEq} / \mathrm{g}$. However fibrillated the specimen, the fixed charge density was never found to be below $0.03 \mathrm{mEq} / \mathrm{g}$. This again agrees with the observations on hip cartilage (Maroudas and others, 1973) and might represent a fraction of the glycosaminoglycans associated more intimately than the rest with the collagen fibres.

It is interesting to note that the fixed charge density of normal cartilage from the patella is considerably lower than that of the femoral head, the mean values differing by some $30 \%$. A lower fixed charge density implies (a) a higher instantaneous deformation of cartilage (Kempson, Muir, Swanson, and Freeman, 1970), and (b) a more rapid fluid loss during load application and a slower recovery (Maroudas, 1973; Maroudas, 1975). Since the pressure on the patella is thought to be at least as high as in the hip (e.g. Maquet, Pelzer, and De Lamotte, 1975), it follows that the patella will normally operate at a lower hydration and larger deformations than the hip. This in turn might result in higher strains in the collagen network at the edges of the loaded areas and might contribute to early damage of the collagen mesh.

Financial support from the British Council and the Medical Research Council is gratefully acknowledged. 


\section{References}

Anderson, C. E., Ludowieg, J., Harper, H. A., AND Engleman, E. P. (1964) J. Bone Jt Surg., 46A 1176 (The composition of the organic component of human articular cartilage)

BOLLET, A. J., AND NANCE, J. L. (1966) J. clin. Invest., 45, 1170 (Biochemical findings in normal and osteoarthritic articular cartilage. II. Chrondroitin sulphate concentration and chain length, water and ash content)

EmERY, I. H., AND MEACHIM, G. (1973) J. Anat., 116, 103 (Surface morphology and topography of patello-femoral cartilage fibrillation in Liverpool necropsies)

Ficat, C. (1974) Sem. Hôp. Paris, 50, 3201 (La dégénerescence du cartilage de la rotule de la chondromalacie à l'arthrose)

Ficat, P. (1973) In 'Les Desequilibres Rotuliens', p. 133. Masson, Paris.

Kempson, G. E., Muir, H., Swanson, S. A. V., AND Freeman, M. A. R. (1970) Biochim. biophys. Acta, 215, 70 (Correlation between stiffness and the chemical constituents of cartilage on the human femoral head)

Maquet, P., Pelzer, G., AND De Lamotte, F. (1975) Acta orthop. belg., 41, 119 (La sollicitation mécanique du genou durant la marche)

Maroudas, A. (1973) In 'Adult Articular Cartilage,' Chapter 4, p. 131. Pitman Medical, London (1975) Biorheology, 12, 233 , AND ThOMAS, H. (1970) Biochim. biophys. Acta, 215, 214 (A simple physicochemical micro method for determining fixed anionic groups in connective tissue)

- Evans, H., AND AlmeIda, L. (1973) Ann. rheum. Dis., 32, 1 (Cartilage of the hip joint. Topographical variation of glycosaminoglycan content in normal and fibrillated tissue)

MeACHIM, G. (1972) Ann. rheum. Dis., 31, 457 (Light microscopy of indian ink preparations of fibrillated cartilage) , AND EMERY, I. H. (1974) Ann. rheum. Dis., 33, 39 (Quantitative aspects of patello-femoral cartilage fibrillation in Liverpool necropsies)

MiLes, J. S., AND EICHELBERGER, L. (1964) J. Amer. Geriat. Soc., 12, 1 